\title{
Bimodality as a signal of Liquid-Gas phase transition in nuclei?
}

\author{
O. Lopez* D. Lacroix, and E. Vient \\ Laboratoire de Physique Corpusculaire \\ IN2P3-CNRS/Université de Caen/ENSICAEN \\ 6, Boulevard Maréchal Juin, F-14050 Caen, France
}

(Dated: November 18, 2018)

\begin{abstract}
We use the HIPSE (Heavy-Ion Phase-Space Exploration) Model to discuss the origin of the bimodality in charge asymmetry observed in nuclear reactions around the Fermi energy. We show that it may be related to the important angular momentum (spin) transferred into the quasi-projectile before secondary decay. As the spin overcomes the critical value, a sudden opening of decay channels is induced and leads to a bimodal distribution for the charge asymmetry. In the model, it is not assigned to a liquid-gas phase transition but to specific instabilities in nuclei with high spin. Therefore, we propose to use these reactions to study instabilities in rotating nuclear droplets.
\end{abstract}

PACS numbers: 24.10.-i,25.70.Mn,64.

Keywords: Heavy-ion induced reactions, Fermi energy, Phase transition, Bimodality

In recent years, the possibility to observe phase transitions in finite, even small systems, has received an increasing interest [1, 2, 3, 4]. In this context, several conceptual questions are addressed, as the extrapolation of thermodynamical properties, or more generally Statistical Mechanics, from finite to infinite systems. For instance, a system with phase transition has discontinuity in the equation of state in the transition region. Such a discontinuity is not present in finite systems but is expected to be replaced by anomalies in specific statistical quantities. One of the possible signature of liquid-gas phase transition in finite systems is the appearance of abnormal fluctuations of the kinetic energy [5] in the microcanonical ensemble, these being at the origin of the so-called negative heat capacity. Equivalently, a bimodal behaviour, i.e. two "bumps" in the energy distribution is expected in the coexistence region, the system being treated canonically. Bimodalities in event distributions is even sometimes promoted as one of the definition of phase transition in finite systems [4, 6]. Indeed, it is related to the anomalous curvature of the entropy or any relevant thermodynamical potential depending on the constraints upon the system (see however 7]). Moreover, several studies have shown that bimodality is rather robust with respect to the introduction of additional constraints [8] on the system or the long-range coulomb force [9]. In this context, nuclei appear as possible candidates to observe liquid-gas phase transition in finite quantum system. Indeed, a large variety of experimental studies 10, 11, 12, 13, 14, 15, 16, 17, 18, 19, 20, 21, 22 reports an accumulation of "evidences" of critical signals. Among them, bimodality in the charge asymmetry of fragments produced in heavy-ion reactions at Fermi energies have been recently reported for the Quasi-Projectiles (QP) isolated in peripheral reactions 21, 22]. This signal is presented as one of the most robust evidence for the liquid-

*Electronic address: lopezo@lpccaen.in2p3.fr gas phase transition in nuclei. However, the extraction of critical signals from nuclear reactions is far from being simple. For example, one expects that a signal initially present at the chemical freeze-out (i.e. when nuclei do not exchange particles anymore) will be largely distorted, or even completely washed out by the secondary decay. This raises the fundamental question of the phase-space explored during the reaction just after fragment formation and its modification due to secondary emission. Recently, the phenomenological HIPSE ${ }^{1}$ model [23] has been developed to address these aspects. In this model, very specific randomness hypotheses are retained to form clusters in the first instants of the reaction, while information on the phase-space explored before and after secondary decay can be accessed without ambiguity. This model, as well as the recently developed version for nucleon-induced reactions (called n-IPSE) 24], has been shown to remarkably reproduce experimental observations.

In this work, we use the HIPSE model to address the question of the origin of the bimodality signal in nuclear reactions. First the experimental protocol used in refs. 21, 22 is recalled. It is applied to events generated with the HIPSE model showing that bimodality is found. Finally, we use the possibility to access the phase-space before the secondary decay to understand the origin of bimodality in the model. To do so, we have generated $10^{6}$ heavy ions collisions for the $\mathrm{Xe}+\mathrm{Sn}$ system at 50 $\mathrm{MeV} /$ nucleon. The full impact parameter distribution, ranging from the grazing to the head-on collisions has been generated. A complete description of the model as well as a discussion of the hypotheses used for cluster formation can be found in refs. [23, 24]. In order to get results directly comparable to those obtained with the INDRA $4 \pi$ array, we have filtered the events and used exactly the same experimental protocol (event sorting) as described in refs. 21, 22]. We first use a completeness

\footnotetext{
1 available at http://lpccaen.in2p3.fr/theorie/theory_lacroix.html
} 


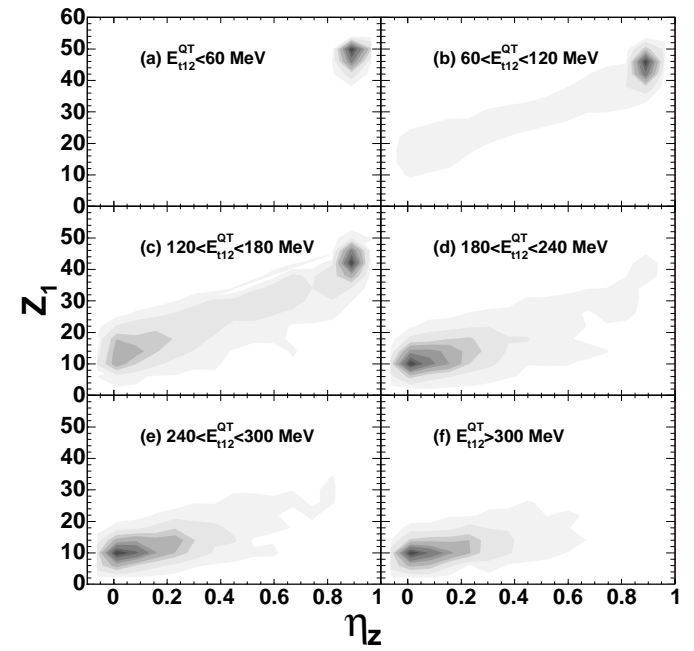

FIG. 1: Correlation between the largest fragment $Z_{1}$ and the charge asymmetry $\eta_{Z}$ for different bins of $E_{t 12}^{Q T}$ (contour levels are in log. scale).

criterion. Here, 'filtered' events, corresponding to the best detection of the QP, are selected ( $80 \%$ of the projectile); this ensures an almost complete detection of the QP products. Due to the forward detection acceptance of INDRA mainly semi-peripheral reactions between 5 to $8 \mathrm{fm}$ are then retained. Complete QP events are finally sorted by using the transverse energy of the light charged particles $(\mathrm{Z}=1,2)$ coming from the Quasi-Target $(\mathrm{QT})$, noted $E_{t 12}^{Q T}$. By doing so, we avoid the obvious autocorrelations between the sorting observable (QT) and the considered system (QP). Note that in the experiment as well as in the simulation, the QP selection has been made by taking fragments with positive center-of-mass velocities [22]; this assumption has been checked with HIPSE and is indeed correct; selecting fragments coming from the true QP source or with positive center-of mass velocities leads to the very same results for this analysis.

In the study of bimodality [21, 22], QT transverse energy is assumed to be indirectly related to the order parameter and is presented as a way of realizing a "canonical" event sorting. Although the transverse energy is intimately correlated to the centrality of the reaction, the latter assumption is, in our opinion, far from being clear because of the associated large mixing of impact parameters. In the following, we will focus on the correlation between the largest and the second largest fragment emitted in the forward center-of-mass hemisphere. We then define the charge asymmetry between the two largest fragments $\eta_{Z}=\left(Z_{1}-Z_{2}\right) /\left(Z_{1}+Z_{2}\right)$ 22]. $Z_{1}$ and $Z_{2}$ are respectively the largest and the second largest fragment charges. Thus, $\eta_{Z}$ is close to 1 for a large asymmetry and it will be the case if an evaporation residue persists after de-excitation. By variance, if $\eta_{Z} \approx 0$, it corresponds to a symmetric fragmentation. Fig. [1 displays the correlation between $Z_{1}$ and $\eta_{Z}$ for different QT transverse energy intervals. Fig. 1(a) shows a single component located at
$\eta_{Z} \approx 1$ and $Z_{1} \approx Z_{\text {proj }}$. This case corresponds to the evaporation residue (ER) of the projectile. In Fig. I(b) to Fig. 廿(f), we observe a different component, located this time at $\eta_{Z} \approx 0$ and $Z_{1} \approx 15$; the corresponding mean fragment multiplicity is here greater than 2, corresponding to the multifragmentation regime (MF). In Fig. 1(c), the correlation clearly exhibits both components, the asymmetric case (ER) and the symmetric one (MF). This coexistence has been assigned to a bimodality signal in the fragmenting nuclear systems 22]. Indeed, by projecting the two-dimensional distribution either on the $x$-axis or $y$-axis, two bumps are observed respectively in the distribution of $\eta_{Z}$ and $Z_{1}$ (not shown here) for the selected intermediate transverse energy. These results are similar to those obtained in the experimental case [22], where a bimodality in $\eta_{Z}$ has been reported for the QP events.

Let us now specify the properties of the two event classes observed in Fig. 1(b) and प(c). The charge partition may mix contributions from quasi-projectile and/or mid-rapidity emissions. Indeed, it results from a possible simultaneous emission leading to a fragmented freezeout configuration followed by a sequential decay of each primary fragments. In the HIPSE model both effects are taken into account and the initial partition before secondary decay can be easily identified. In particular, the number of sources contributing to the bimodality is of major interest. By tracing back the origin of clusters in HIPSE during the decay, we have observed that the bimodality signal displayed in Fig. 1 is dominated by events where a single source is formed in the forward center-of-mass hemisphere, this source corresponding indeed to the excited QP. To get a deeper insight on the origin of bimodality in HIPSE, it is necessary to clearly identify the phase-space explored by the QP before de-excitation for the two event classes. Here, we concentrate on Fig. प(c) and we will refer to ER for events with $\eta_{Z}>0.8$ and MF for $\eta_{Z}<0.2$. Fig. 2 presents respectively from top to bottom the correlation between the size, the thermal energy, the transferred angular momentum and the impact parameter for ER (left) and for MF (right). The first remarkable aspect appearing in top panels of Fig. 22 is that the source sizes are not significantly different between ER $\left(Z_{Q P} \approx 50\right)$ and $\mathrm{MF}$ case $\left(Z_{Q P} \approx 45\right)$ and the two distributions strongly overlap. By contrast, the thermal energy $E_{t h}$ is much higher in the $\operatorname{MF}\left(E_{t h} / A \approx 4 M e V\right)$ than in the ER case $\left(E_{t h} / A \approx 1.5 \mathrm{MeV}\right)$. It is worth noticing that such a result is at variance with a geometrical scenario like 'abrasion-ablation' models where such an increase of thermal energy is accompanied by a strong decrease of the QP size [25]. Indeed, in the HIPSE model, while the QP and QT are initially formed using geometrical arguments, the abrasion picture is partially (or even completely) relaxed by allowing nucleon exchange and by the strong reorganisation due to Final State Interaction (FSI) [26]. For the beam energy considered here $(50 \mathrm{~A} \cdot \mathrm{MeV})$, an important exchange of particle between 


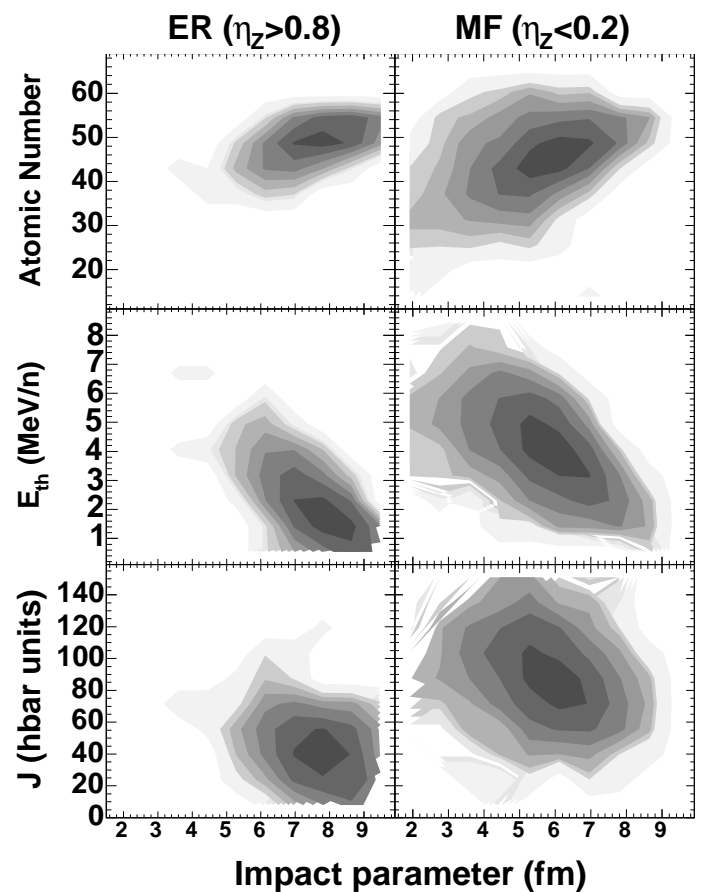

FIG. 2: Correlation between the atomic number (top row), the thermal energy (middle) and spin (bottom) of the QP source for events of Fig. 11(c). The left column corresponds to the ER case and the right to MF case (contour levels are in log. scale).

the target and projectile should also be accompanied by a large transfer of orbital into intrinsic angular momentum (spin). This is indeed confirmed in bottom part of Fig. 2 where the correlation between the QP angular momentum (noted $J$ ) and the impact parameter is displayed. Again, we observed that while ER corresponds in average to $J \approx 30 \hbar$, in MF much higher angular momenta are obtained $(J \approx 80 \hbar)$. Consequently, the two classes of events associated to bimodality are issued from the de-excitation of a single-source with more or less the same mass but rather different initial conditions in term of thermal energy and angular momentum. Note that here, the de-excitation is performed in both cases using the statistical sequential decay model of ref. [27]. Therefore, in HIPSE, the appearance of the two contributions in Fig. 1 is a direct consequence of the statistical decay accounting for the initial properties of the QP. To go further, we have performed statistical simulations for a given nucleus $(Z=50)$ by varying the initial thermal energy and spin. Fig. 3 shows the corresponding $\eta_{Z}$ distributions. If the spin is set to zero (top row), we see that the higher the thermal energy, the smaller the charge asymmetry is, but we never obtain a crossover to small values of $\eta_{Z}$. The situation is clearly different when the spin is changed. We see in Fig. [3 that at low spin $(J=0 \hbar$ and $30 \hbar)$ and high spin $(J=90 \hbar)$ only a single contribution exists respectively corresponding to high and low $\eta_{Z}$ values. In opposite, for spin $J=60-70 \hbar$, we observe a

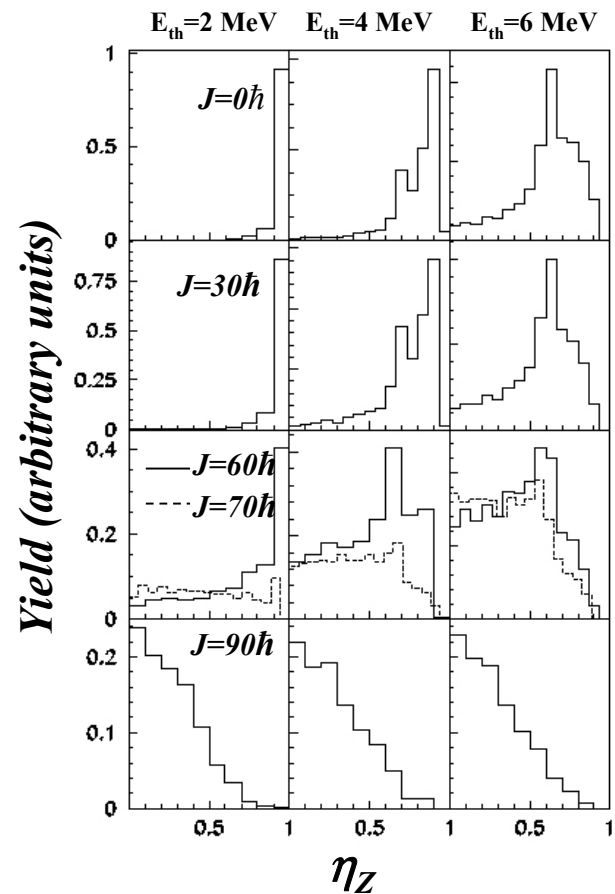

FIG. 3: $\eta_{Z}$ distributions for the statistical de-excitation of an ${ }^{120} \mathrm{Sn}$ nucleus for different initial thermal energies $E_{t h}$ (from left to right) and spins $J$ (from top to bottom).

sudden transition where both contributions coexist. This transition appears to be almost independent on the thermal energy. Therefore, the bimodality observed in Fig. 3 is related to a transition governed by the spin transferred in the collision, but is not of the liquid-gas type as concluded in [22].

The physical origin of this bimodal behaviour in the statistical decay can be inferred from the value of the spin associated to the transition. Indeed, it corresponds approximately to the limit of stability of a nucleus with $Z \approx 50$ against prompt fission 28]. At that point, the fission barrier height becomes comparable to the energy of the least-bound particle and the nucleus cannot resist to the deposited spin. In the statistical model this corresponds to a sudden opening of decay channels leading to the low $\eta_{Z}$ contribution. It is worthwhile to mention that the description of such instability through a statistical model is certainly an approximation. Actually, in a complete dynamical description of this instability we do expect that the system breaks almost at the same time as it is formed. In the HIPSE model, the system is assumed to be formed and then explore statistically accessible final configurations; it is certainly a too simplistic picture and calls for further theoretical developments. In order to check our interpretation, we have replaced the standard HIPSE deexcitation stage by a statistical multifragmentation model (SMM 29], using a freeze-out volume of 3 times the normal density), i.e. we used the HIPSE phase space before decay as an event-by-event input to SMM. We present in Fig. 4 the correlation between the largest 


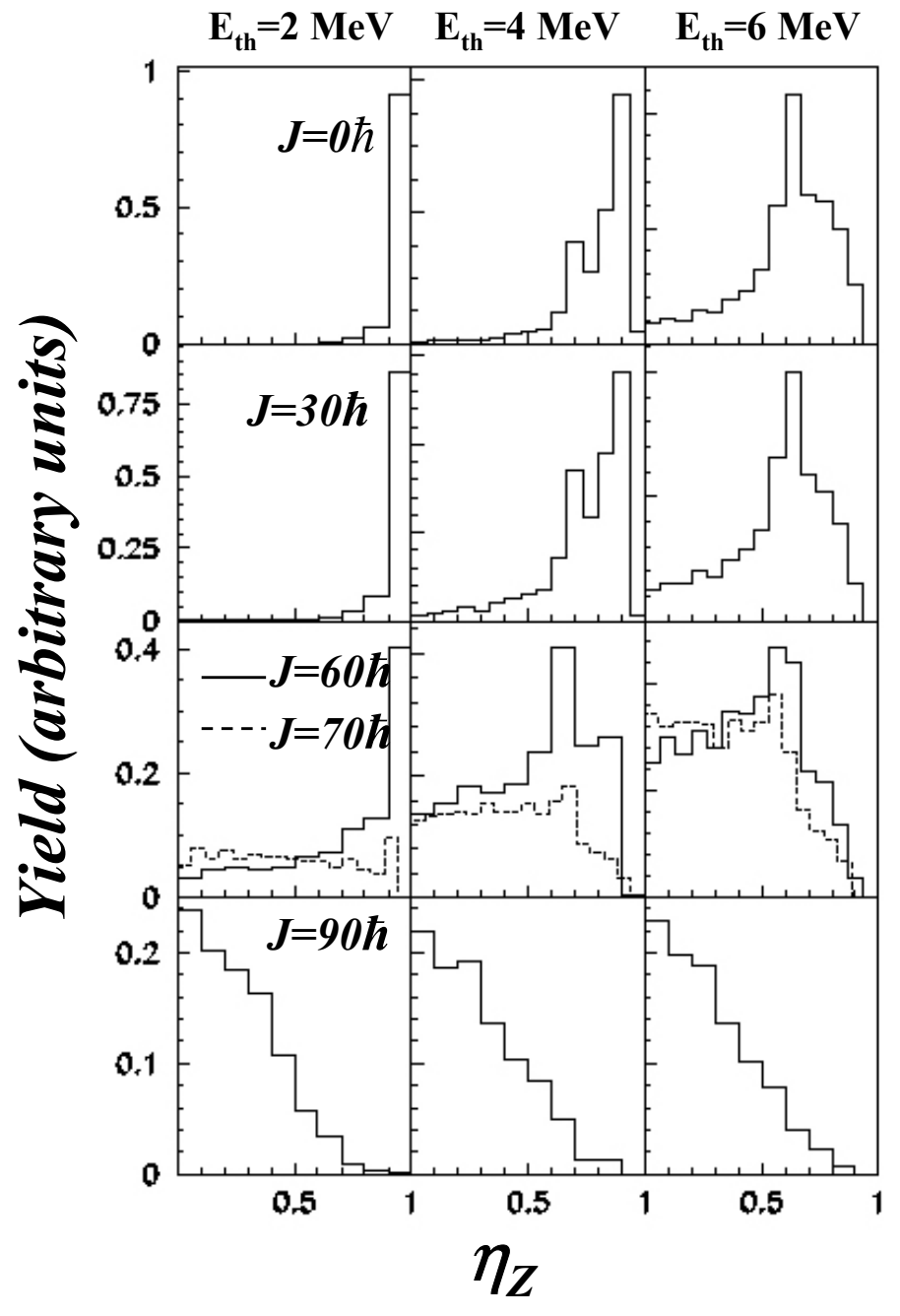

and the second largest fragment obtained with HIPSE (left panel) and the new decay provided by SMM (right panel). They display very similar features indicating that both scenarii are compatible with bimodality. Therefore, we need additional observables, for instance related to the kinematical properties. Indeed, first investigations on experimental data indicate that the angular distributions of the emitted fragments are changed depending on the charge asymmetry selection and this could be attributed to different spin deposition into the decaying nucleus [30]. However, angular distributions need to be used very cautiously because of the difficulty to assess the true origin of fragments or particles (mid-rapidity, preequilibrium, statistical emissions); it could blur the spin effect on the angular distributions 31]. Such a study, comparing quantitatively HIPSE predictions to experimental data for QP deexcitation, will be reported in a forthcoming article. Nevertheless, following the HIPSE scenario which has provided a good reproduction of a large number of experimental observations, including angular distributions for LCP and fragments 23, 32], we conclude that nuclear systems close or beyond their limit of resistance with respect to spin deposition, can be formed, leading then to a bimodal behavior. Therefore, heavy-ion induced reactions might be a tool to study the emergence of shape bifurcation associated to high spin, called the Jacobi sequence 33]. This is a very interesting aspect which has not been explored in this context. If this interpretation is confirmed experimentally, instead of a liquid-gas phase transition, we may have a signature of the so-called Jacobi transition 34], which is related to a second-order phase transition in the continuous limit and has also its equivalent in astrophysical context 35].
FIG. 4: Correlation between the largest $\left(Z_{1}\right)$ and the 2nd largest fragment $\left(Z_{2}\right)$ obtained with the standard HIPSE (left) and SMM decay (right). Both simulations are made with the same set of $\left(\mathrm{Z}, \mathrm{A}, \mathrm{E}^{*}\right)$ given by the preequilibrium stage of HIPSE (contour levels are in log. scale).
[1] D.H.E. Gross, Microcanonical thermodynamics: Phase transition in Small systems, vol. 66, Lecture Notes in Physics, World Scientific, Singapour, (2001).

[2] J. Richert and P. Wagner, Phys. Rep. 350, 1 (2001)

[3] Ph. Chomaz, M. Colonna and J. Randrup, Phys. Rep. 389, 263 (2004)

[4] Ph. Chomaz and F. Gulminelli, Nucl. Phys. A749, 3 (2005)

[5] Ph. Chomaz and F. Gulminelli, Nucl. Phys. A647, 153 (1999)

[6] Ph. Chomaz and F. Gulminelli, Physica A330, 451 (2003)

[7] H. Touchette, ArXiv:cond-mat/0503029

[8] F. Gulminelli, Ph. Chomaz, Nucl. Phys. A734, 581 (2004)

[9] F. Gulminelli, Ph. Chomaz, Al. H. Raduta, and Ad. R. Raduta, Phys. Rev. Lett. 91, 202701 (2003)
[10] M.F. Rivet et al, Nucl. Phys. A749, 73 (2005)

[11] M. D'Agostino et al, Nucl. Phys. A749, 55 (2005)

[12] M. D'Agostino et al., Phys. Lett. B 473, 219 (2000)

[13] J. B. Elliott et al., Phys. Rev. Lett. 88, 042701 (2002)

[14] G. Tabacaru et al., Eur. Phys. J. A 18, 103 (2003)

[15] J. D. Frankland et al., Nucl. Phys. A 689, 940 (2001)

[16] M. B. Tsang, W. A. Friedman, C. K. Gelbke, W. G. Lynch, G. Verde and H. Xu, Phys. Rev. Lett. 86, 5023 (2001)

[17] W. Trautmann, Nucl. Phys. A 685, 233 (2001)

[18] R. Wada et al., Phys. Rev. C 69, 044610 (2004)

[19] J. B. Natowitz et al., Phys. Rev. C 65, 034618 (2002)

[20] Y.G. Ma et al, Nucl. Phys. A749, 106 (2005)

[21] B. Tamain et al, Proceedings of the IWM Meeting, Caen, France, 83 (2003)

[22] M. Pichon et al, Nucl. Phys. A749, 93 (2005)

[23] D. Lacroix, A. Van Lauwe, and D. Durand, Phys. Rev. 
C69, 054604 (2004)

[24] D. Lacroix, V. Blideanu, and D. Durand, Phys. Rev. C71, 024601 (2005)

[25] J.-J. Gaimard and K.-H. Schmidt, Nucl. Phys. A531, 709 (1991)

[26] D. Lacroix, A. Van Lauwe, and D. Durand, Proceedings of the IWM Meeting, Caen, France, 58 (2003)

[27] D. Durand, Nucl. Phys. A451, 266 (1992)

[28] P. Frobrich and R. Lipperheide, Theory of Nuclear Reactions, Clarendon Press, Oxford (1996)

[29] J.P. Bondorf, A.S. Botvina, A.S. Iljinov, I.N Mishustin and K. Sneppen, Statistical Multifragmentation of Nuclei,
Phys. Rep. 257, 133 (1995)

[30] J.C. Steckmeyer, Z. Sosin, K. Grotowski, P. Pawlowski, ArXiv: nucl-ex/0504025

[31] J.C. Steckmeyer et al, Nucl. Phys. A686, 537 (2001)

[32] A. Van Lauwe, D. Lacroix and D. Durand, Proceedings of the IWM Meeting, Caen, France, 62 (2003).

[33] P. Ring and P. Schuck, The Nuclear Many-Body Problem, Spring-Verlag, New-York (1980), Chap. 1

[34] A. Maj et al, Nucl. Phys. A731, 319 (2004)

[35] D.M. Christodoulou, D. Kazanas, I. Schlosman and J.E. Tohline, ArXiv: astro-ph/9505008 